\title{
A design procedure for an acoustic mirror providing dual reflection of longitudinal and shear waves in Solidly Mounted BAW Resonators (SMRs)
}

\author{
$\underline{\text { S.Jose }}^{\underline{I}}$, A.B.M.Jansman ${ }^{2}$ and R.J.E. Hueting ${ }^{I}$ \\ ${ }^{1}$ MESA+ Institute for Nanotechnology, Semiconductor Components, University of Twente, Enschede (NL) \\ ${ }^{2}$ Research, NXP Semiconductors Eindhoven (NL)
}

\begin{abstract}
The quality factor of the traditional Solidly Mounted Resonator (SMR) is limited by substrate losses, as the traditionally employed acoustic mirror reflects longitudinal waves but not shear waves. Modern mirrors do reflect both waves, but design rules for such mirrors have not been published so far. We propose a systematic design procedure derived from optics leading to a novel embodiment for the acoustic mirror which effectively reflects both longitudinal and shear waves. This method can be applied for the acoustic mirror design for any given material combination. An analytical model is presented; its agreement with FEM simulations is good. With the optimized design, we can obtain a minimum transmission for longitudinal and shear waves of $-25 \mathrm{~dB}$ and $-20 \mathrm{~dB}$ at resonance frequencies for longitudinal and shear waves, respectively, for various reflector material combinations.
\end{abstract}

Keywords - Bulk Acoustic Wave (BAW), Solidly Mounted Resonator (SMR), Acoustic mirror, Stop-band theory, Transmission curve.

\section{INTRODUCTION}

$\mathrm{T}$ hin-film bulk-acoustic-wave (BAW) devices are used for RF selectivity in mobile communication system and other wireless applications. Currently surface-acoustic-wave (SAW) is the preferred technology for RF filters. However thin-film BAW devices have several advantages as they are remarkably small in size, have better power handling abilities and better temperature coefficients leading to more stable operation [1]. From a practical point of view SAW filters have considerable drawbacks beyond $2 \mathrm{GHz}$ whereas BAW devices up to 20 $\mathrm{GHz}$ have been demonstrated [2]. BAW is expected to supersede SAW as the technology of choice in many applications over the next few years as they have now evolved in performance beyond SAW and can be manufactured in a very cost competitive way using standard IC technology.

BAW technology is commercially available for US-PCS $(1.85 \mathrm{GHz}-1.91 \mathrm{GHz})$ applications. Transmit and receive bands of the US-PCS standard are close in frequency [3]. This demands BAW resonators which constitute the narrow band filters to be nearly loss-free. Hence one of the important goals of the BAW community is to come up with high $Q$ resonators for RF filters by minimizing the losses [4-8].
The quality factor of traditional Surface Mounted Bulk Acoustic Wave Resonators (SMRs) is generally limited by substrate losses [9-12]. This is due to the fact that the conventional quarter wave acoustic mirror employed in SMRs reflects only the longitudinal acoustic waves and not the shear waves. In order to obtain SMRs with high quality factors, the mirror should effectively reflect both waves. The necessity for reflection of both types of waves was recognized earlier and the influence of shear waves on Q factor is reviewed in [11]. Some optimized stacks have been reported for specific material combinations [9-10] based on numerical calculations. But to our knowledge no systematic design procedure with a solid theoretical background has been reported. We present a procedure, derived from Optics [13], to design stacks that effectively reflect both longitudinal and shear waves. The salient feature of this method is that it can be applied for any material combination.

\section{ACOUSTIC MIRROR IN SMRs}

The acoustic mirror design is critical for the performance of SMRs. Fig. 1 shows a typical SMR structure in which the piezoelectric resonator is isolated from the substrate using an acoustic mirror [4]. The $1260 \mathrm{~nm}$ piezoelectric layer is formed by aluminum-nitride (AlN).

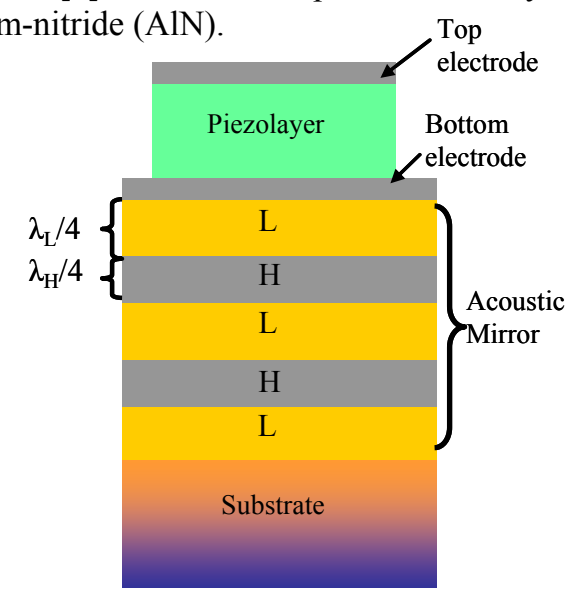

Figure 1. Solidly Mounted BAW Resonator (SMR) with quarter-wave thick acoustic mirror. $L$ and $H$ indicate layers having a light and heavy acoustical impedance, respectively.

Conventionally these mirrors are optimized to reflect longitudinal acoustic waves. The best reflection is obtained if the reflector consists of alternating layers of low and high acoustic impedances, and when the ratio of these impedances 
is maximal [5]. For optimal reflection, the layer thickness $t_{\mathrm{n}}$ of each layer $n$ is a quarter of the wavelength for longitudinal waves $\lambda_{\text {long }}$ at resonance frequency.

$$
t_{n}=\frac{\lambda_{\text {long }}}{4} \text {. }
$$

The quarter-wave mirror designed using eqn. (1) reflects only longitudinal waves and shear waves are not optimally reflected by this stack. This can be explained by the fact that the shear wave velocity is usually about half of the longitudinal wave velocity [9]. By employing then the transmission line model for the stack (see e.g. [4]) and with the approach of mirror reflectivity [12], the transmission curve as a function of frequency of such a mirror is plotted. Fig.2 shows the transmission curve of a nine layer $\mathrm{Ta}_{2} \mathrm{O}_{5} / \mathrm{SiO}_{2}$ $(762 \mathrm{~nm} / 588 \mathrm{~nm})$ quarter-wave mirror optimized at $1.88 \mathrm{GHz}$. It is seen from the graph that minimum transmission of longitudinal waves corresponds to maximum transmission for shear waves (and vice versa). This would result in unacceptably low quality factors. Here we present a solution for this problem using some theories derived from optics which is presented in section III.

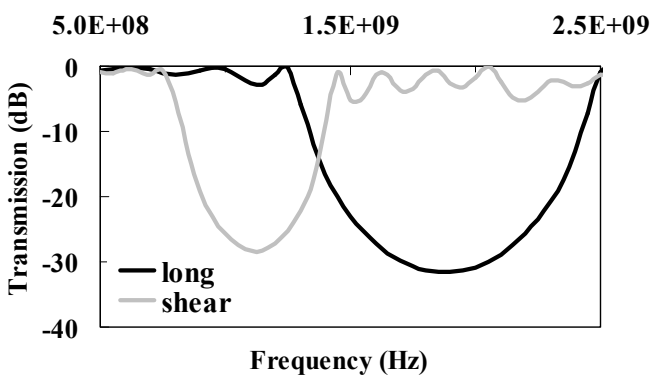

Figure 2. Transmission curves of longitudinal and shear waves of a nine layer $\mathrm{Ta}_{2} \mathrm{O}_{5} / \mathrm{SiO}_{2}$ quarter-wave reflector stack optimized for minimum transmission for longitudinal waves.

\section{DESIGN PROCEDURE}

It may be recalled that the acoustic mirror is essentially a periodic structure and the goal of this design approach is to minimize the transmission for two types of waves through such a structure at the resonance frequency. The stop-band theory for a periodic structure is well defined in [13]. Optics of thin films defines a stop-band as high reflectivity region (or equivalently low transmission region) which is a characteristic feature of any periodic structure. The cardinal point of the stop-band is defined as the point of maximum reflection in this region. Cardinal points can be calculated explicitly for layer stacks, with a bi-layer unit cell. The cardinal points of the stop bands can be found by solving the equation

$\varphi_{\mathrm{L} . \mathrm{x}}+\varphi_{\mathrm{H} . \mathrm{x}}=(1+c) \varphi=\mathrm{n} \pi, \mathrm{n}$ integer

where $\varphi_{\mathrm{L} . x}$ and $\varphi_{\mathrm{H} . x}$ are the phase angles in low index layer and high (refractive) index layer of the basic two-layer dielectric stack (bi-layer). The suffice in L. $x$ and H. $x$ indicates that $x$ could be longitudinal or shear waves. $c$ is the detuning ratio defined as the ratio of $\varphi_{\mathrm{H}}$ to $\varphi_{\mathrm{L}}$. For later purposes, $c$ is introduced for translation from optics to acoustics. The solution for $\varphi$ gives the cardinal points and this can be extended for $\mathrm{N}$ number of identical bi-layers.

Depending on the values of $\mathrm{n},[13]$ classifies the dielectric design units into $\mathrm{HnL}$ configurations having $\mathrm{n}$ solutions for cardinal points. Let us take the case of H2L (HLL) configuration for longitudinal waves ( $x=$ long) having two solutions $n=1$ and $n=2$ (see eqn. (2)). For $n=1$ and $n=2$ the cardinal points are at $\pi /(1+\mathrm{c})$ and $2 \pi /(1+\mathrm{c})$, respectively. We want them to occur at $\pi / 2$ because we want the reflection of longitudinal and shear waves to reflect at single resonance frequency $f_{\mathrm{R}}$. A phase compensation of $\varphi_{1}{ }^{\prime}=\pi / 2-\pi /(1+\mathrm{c})$ and $\varphi_{2}{ }^{\prime}=2 \pi /(1+\mathrm{c})-\pi / 2$ is required. This is then translated into compensating thicknesses $h_{\mathrm{L}}$ and $h_{\mathrm{H}}$.

$h_{\mathrm{L}}=\frac{\varphi_{1}^{\prime}}{2 \pi} \lambda_{\text {L.long }}=\frac{\frac{\pi}{2}-\frac{\pi}{1+c}}{2 \pi} \lambda_{\text {L.long }}=\left(\frac{1}{4}-\frac{1}{2+2 c}\right) \lambda_{\text {L.long }}$,
$h_{\mathrm{H}}=\frac{\varphi_{2}^{\prime}}{2 \pi} \lambda_{\text {H.long }}=\frac{\frac{2 \pi}{1+c}-\frac{\pi}{2}}{2 \pi} \lambda_{\text {H.long }}=\left(-\frac{1}{4}+\frac{1}{1+c}\right) \lambda_{\text {H.long }}$.

where $\lambda_{\text {L.long }}$ and $\lambda_{\text {H.long }}$ are the quarter wavelengths for longitudinal waves in low impedance and high impedance layers respectively. Note that $\mathrm{c}$ in stop-band theory was defined as the phase ratio of two layers while now we define $\mathrm{c}$ to be the average velocity ratio of the waves according to:

$$
c=\frac{\frac{v_{\text {Llong }}}{v_{\text {Lshear }}}+\frac{v_{\text {Hlong }}}{v_{\text {Hshear }}}}{2},
$$

where $v_{\mathrm{y} \cdot \text { long }}$ and $v_{\mathrm{y} \text {.shear }}$ are the velocities of longitudinal and shear waves, respectively, for $y=\mathrm{L}$ (low impedance), $\mathrm{H}$ (high impedance). Alternative ways to define c are also possible, but these are not discussed in this work. This whole exercise is easily collected into one formula for the final thicknesses $t_{\mathrm{L}}$ and $t_{\mathrm{H}}$.

$$
\begin{aligned}
& t_{\mathrm{L}}=\frac{\lambda_{\mathrm{L} . \text { long }}}{4}-h_{\mathrm{L}}=\lambda_{\mathrm{L} \text {.long }}\left(\frac{1}{4}-\frac{1}{4}+\frac{1}{2+2 c}\right)=\frac{1}{2+2 c} \lambda_{\text {L.long }}, \\
& t_{\mathrm{H}}=h_{\mathrm{H}}-\frac{\lambda_{\mathrm{H} . \text { long }}}{4}=\lambda_{\text {H.long }}\left(-\frac{1}{4}+\frac{1}{1+c}+\frac{1}{4}\right)=\frac{1}{1+c} \lambda_{\mathrm{H} . \text { long }} .
\end{aligned}
$$

The optimized acoustic mirror with thicknesses calculated by eq. (6) and (7) effectively reflects both longitudinal and shear waves as seen in Fig. 4 (Section IV). One can further optimize the reflection for both types of waves by introducing a spacer [14]. The spacer layer was further tuned for performance improvement and the improved layer configuration of acoustic mirror is (LHL) $(0.5 \mathrm{H} 2 \mathrm{~L} 0.5 \mathrm{H})$ (LHL) which is shown in Fig. 3. Note that the figures in the configuration indicate the relative thickness, e.g. $0.5 \mathrm{H}$ indicates $0.5 \cdot \lambda_{\text {H.long }}$.

The optimized acoustic mirror has nine alternate layers of $\mathrm{SiO}_{2}$ and $\mathrm{Ta}_{2} \mathrm{O}_{5}$. The total number of layers can be increased until the reflection is sufficiently large in magnitude to give the desired resonator response. In addition, the impedance ratio between adjacent layers determines the magnitude of 
reflection. It is found from the simulations that a reflector stack with odd number of layers has a better performance.

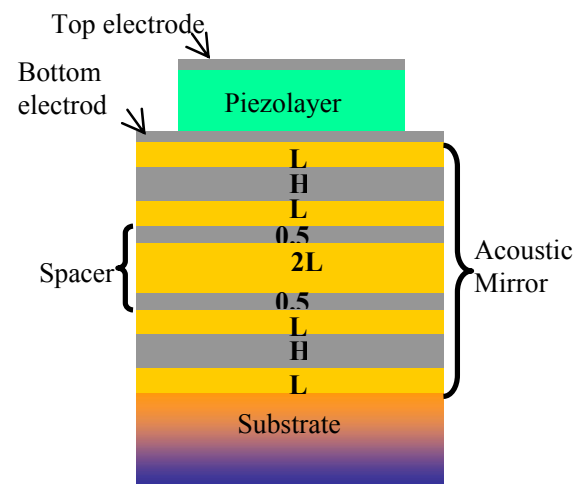

Figure 3. Solidly Mounted BAW Resonator (SMR) with the optimized acoustic mirror in an improved configuration. Layer thicknesses are summarized in Table 1.

\section{DisCUSSION OF RESULTS}

The design theory described in Section III has been verified both with analytical and Comsol FEM simulations [15]. They are found to be in good agreement for different material combinations investigated.

\section{A. Mirror Transmission curves}

The results from the one-dimensional analytical model are presented in Fig. 4. It shows the transmission curve of the optimized reflector stack with and without the spacer. It can be seen from the figure that using the optimized thicknesses calculated by the new design procedure has minimized the transmission at $1.88 \mathrm{GHz}$ and using these thicknesses in the (improved) spacer configuration brings the transmission bands into coincidence.

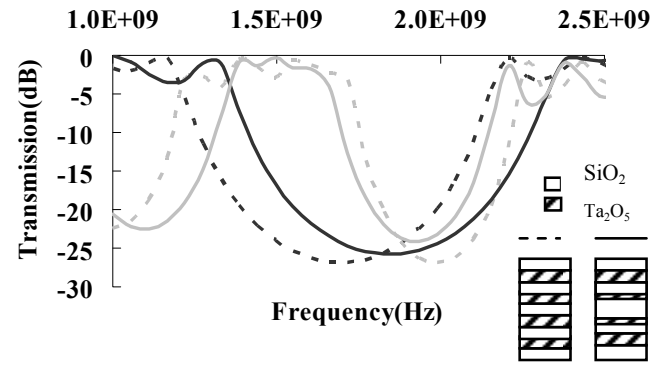

Figure 4. Transmission curves of a nine layer $\mathrm{Ta}_{2} \mathrm{O}_{5} / \mathrm{SiO}_{2}$ optimized reflector stack based on the new design procedure with and without the spacer layer. Black lines indicate longitudinal and grey indicate shear waves. Solid lines represent the configuration with the spacer layer and dashed lines represent the ones without the spacer layer.

\section{B. Displacement profile}

The results were also confirmed with the displacement profiles of the waves in the device. The displacement profiles comparison of longitudinal and shear waves from Comsol and analytical simulations for a conventional quarter wave mirror and the ones with optimized thicknesses in the improved configuration is shown in Fig. 5. It can be seen from the figure that with the new optimized stack, shear wave displacement in the device is getting minimized without longitudinal wave displacement getting much affected. Acoustic field distribution for longitudinal waves is mainly concentrated in the top oxide layers and remains almost the same in the optimized stacks. However shear waves penetrate deeper into the mirror till the middle spacer layer. In other words, the spacer layer seems to act as a barrier collecting the shear waves and hence preventing it from further leaking into the layers below.

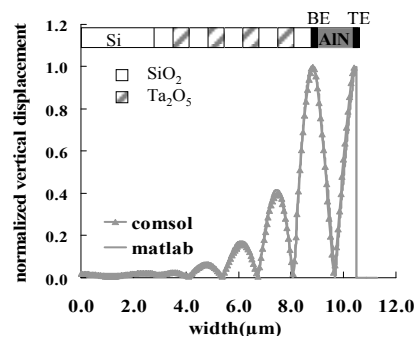

(a)

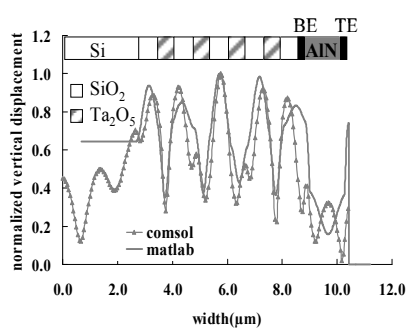

(c)

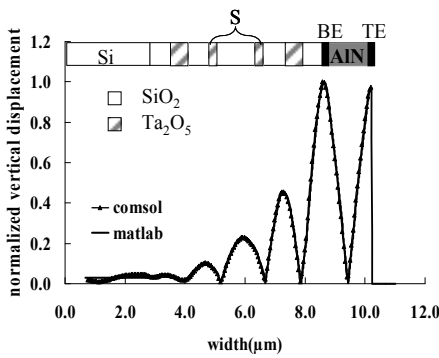

(b)

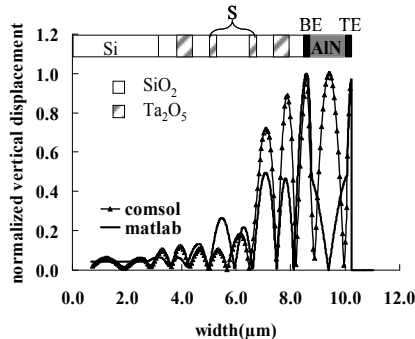

(d)
Figure. 5. Displacement profile of (a) longitudinal waves in conventional stack (b) longitudinal waves in optimized stack (c) shear waves in conventional stack and (d) shear waves in optimized stack. The SMR structure is represented on the top of each diagram. BE - Bottom Electrode, TE - Top Electrode and $\mathrm{S}$ - spacer. Curves are normalized with the maximum value of displacement in each case ((a) $5.7 \cdot 10^{-9} \mathrm{~m} \mathrm{(b)}$ $\left.5.6 \cdot 10^{-9} \mathrm{~m}(\mathrm{c}) 5.2 \cdot 10^{-11} \mathrm{~m}(\mathrm{~d}) 1.7 \cdot 10^{-10} \mathrm{~m}\right)$.

\section{Material Combinations}

To demonstrate the usefulness of the scheme, the new design procedure was applied for different material combinations such as $\mathrm{W} / \mathrm{SiO}_{2}, \mathrm{SiOC} / \mathrm{Ta}_{2} \mathrm{O}_{5}, \mathrm{SiOC} / \mathrm{W}, \mathrm{SiOC} / \mathrm{Pt}, \mathrm{SiOC} / \mathrm{TiN}$. In all the cases, the optimized layer stacks in the new configuration show efficient reflection of both longitudinal and shear waves. Fig.6 shows the curves demonstrated for different material combinations. The reason for much lower transmission coefficients compared to Fig. 4 is attributed to the use of higher ratios of heavy and light acoustic impedances.

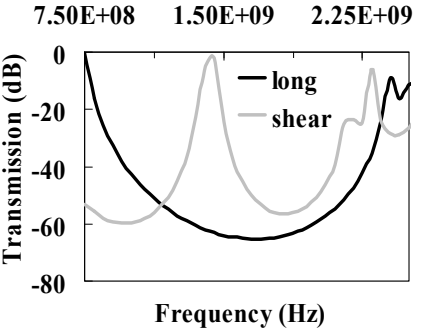

(a)

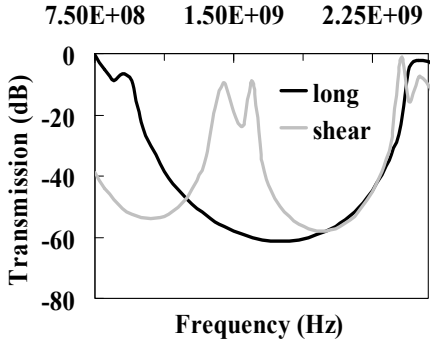

(b)
Figure 6. Transmission curves of a nine layer optimized reflector stack of (a) $\mathrm{W} / \mathrm{SiO}_{2}$ and (b) $\mathrm{SiOC} / \mathrm{Ta}_{2} \mathrm{O}_{5}$. In both the cases, the transmission of longitudinal and shear waves are well below $-55 \mathrm{~dB}$. 


\section{Impedance curves}

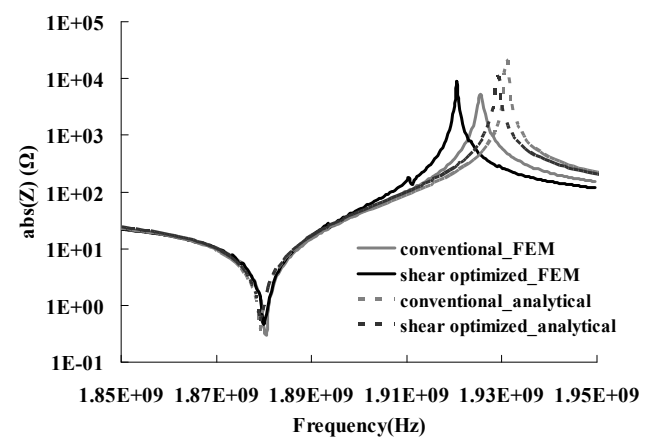

Figure.7. Simulated and analytical impedance vs. frequency of a resonator with a conventional quarter-wave reflector and an optimized reflector stack based on the new design procedure. (Electrode area: 140x140 $\mu \mathrm{m}^{2}$ ).

A comparison of the impedance curves of the resonators is also made to study the performance improvement. Fig.7 shows the simulated impedance vs. frequency of a resonator with a conventional quarter-wave reflector and the optimized reflector obtained from analytical and Comsol simulations. The analytical and the FEM results show the same trend although there is a shift in the anti-resonant frequency. This can be attributed to the fact that the analytical model is truly 1D and the FEM simulations are 2D in which the top electrode has a finite dimension. Also, the shear-optimized curve obtained from FEM simulations shows stronger spurs indicating that the acoustic energy is better confined in the new reflector than in the conventional quarter-wavelength reflector. Hence an improvement in acoustical Q is expected. FEM simulations show an improvement of Q from 960 to 1480 when calculated using the phase derivative method [5].

\section{E. Sensitivity Analysis}

Analogue to an earlier report [16], the dependence of the layer thickness on mirror transmission has been investigated. The thickness of the layer was varied individually per nanometer, percentage thickness and per ten percentage thickness. Transmission of longitudinal and shear waves were noted. The results are shown in Table1.

Table1.Simulated sensitivities of transmission vs. layer thickness for a nine layer $\mathrm{Ta}_{2} \mathrm{O}_{5} / \mathrm{SiO}_{2}$ optimized acoustic mirror at $1.88 \mathrm{GHz}$

\begin{tabular}{|c|c|c|c|}
\hline Layer & $\begin{array}{l}\text { Thickness } \\
(\mathrm{nm})\end{array}$ & $\begin{array}{l}\text { Sensitivity }(\mathrm{dB}) / \\
10 \% \text { thickness } \\
\text { (Longitudinal) }\end{array}$ & $\begin{array}{l}\text { Sensitivity }(\mathrm{dB}) / \\
10 \% \text { thickness } \\
\text { (shear) }\end{array}$ \\
\hline TEOS & 559 & 0.104 & -0.204 \\
\hline $\mathbf{T \mathbf { a } _ { 2 }} \mathbf{O}_{5}$ & 862 & -0.871 & 0.569 \\
\hline TEOS & 559 & 0.233 & -0.258 \\
\hline $\mathbf{T a}_{2} \mathbf{O}_{5}$ & 431 & 0.258 & -0.292 \\
\hline TEOS & 1118 & -0.727 & 1.286 \\
\hline $\mathrm{Ta}_{2} \mathrm{O}_{5}$ & 431 & 0.264 & -0.207 \\
\hline TEOS & 559 & 0.249 & 0.001 \\
\hline $\mathrm{Ta}_{2} \mathrm{O}_{5}$ & 862 & -0.815 & 1.637 \\
\hline TEOS & 559 & 0.157 & 0.536 \\
\hline
\end{tabular}

It is noted that the transmission for longitudinal waves appears to be most sensitive to the thickness of the first Ta2O5 layer and least sensitive to that of the first TEOS layer. In the case of shear waves, it does not follow a rigid trend, though in many cases shear transmission is mainly sensitive to the thickness of the middle spacer layer. This can be explained by the discussion in section IV.B; the longitudinal and shear waves are primarily confined till the top two layers, and the spacer layer, respectively. Any change in these layers affects the penetration depth. An increase in 10 percentage thickness only can bring a change of $\pm 1 \mathrm{~dB}$. Therefore, the thickness tolerance of the optimized acoustic mirror can be defined as \pm 10 percentage thickness. Note that the sensitivities in the quarter wave stack were lower for the shear waves. This is because of the deeper, and more uniform, penetration (Fig. 5).

\section{CONCLUSIONS}

A new design procedure for an acoustic mirror providing dual reflection of longitudinal and shear waves in solidly mounted BAW resonators has been proposed. The design procedure has been implemented in both analytical and FEM models. A comparison of transmission characteristics and displacement profile is carried out and results are found to be impressive. The procedure has been demonstrated for different material combinations and in all the cases a minimum transmission of $-25 \mathrm{~dB}$ and $-20 \mathrm{~dB}$ at resonance frequencies were obtained for longitudinal and shear waves respectively. Adaptability of this scheme makes it a potential design guide-line for the devices with minimized acoustic losses into the substrate.

\section{ACKNOWLEDGMENT}

The authors gratefully acknowledge the financial support by the Dutch Ministry of Economic Affairs in the framework of the Point one project MEMSLand. We also thank Jaap Ruigrok (NXP Research, Eindhoven) for the valuable Comsol discussions.

\section{REFERENCES}

[1] H. P. Loebl et al, "RF bulk acoustic resonators and filters," Kluwer Journal of Electroceramics, 12, pp.109-118, 2004.

[2] K. M. Lakin et al., "Bulk acoustic wave resonators and filters for applications above $2 \mathrm{GHz}$," IEEE MTT-S Int. Symp. Digest, pp. 1487-1490, June 2002.

[3] E.Schmidhammer et al., "Design flow and methodology on the design of BAW components," IEEE MTT-S Int. Symp. Digest, pp. 233-236, June 2005.

[4] K.M.Lakin et al., "High-Q Microwave acoustic resonators and filters," IEEE Trans. Microwave Theory and Techniques, 41(12), pp. 2139-2146, 1993

[5] F.Z.Bi et al., "Bulk acoustic wave RF technology," IEEE Microwave magazine, pp. 65-80, October 2008 .

[6] R. Aigner, "Bringing BAW Technology into Volume Production:The Ten commandments and the seven deadly sins," IEEE Int. Symp. Acoust. Wave. Dev. for Future Mobile Communication Syst, 2007.

[7] J.-W. Lobeek et al., "High-Q BAW resonator on $\mathrm{Pt} / \mathrm{Ta} 2 \mathrm{O} 5 / \mathrm{SiO} 2$-based reflector stack," Proc. IEEE Microwave Symposium, pp.2047-2050, 2007.

[8] R. Strijbos et al., "Design and characterization of High-Q Solidly-Mounted Bulk Acoustic Wave Filters," Proc. IEEE Electronic components and technology conference, pp.169-174, 2007.

[9] S. Marksteiner et al., "Optimization of Acoustic Dispersion for high performance thin film BAW resonators," Proc. IEEE Ultrasonics Symposium, pp.1175-1178, 2005.

[10] S. Marksteiner et al., Acoustic Reflector for a BAW resonator providing specified reflection of both shear wave and longitudinal waves, US patent: 006933807B2.

[11] J.Kaitila, "Review of wave propagation in BAW thin film devices progress and prospects," Proc. IEEE Ultrasonics Symposium, pp.120-129, 2007.

[12] S. Marksteiner et al., "Optimization of Acoustic mirrors for Solidly Mounted BAW resonators," Proc. IEEE Ultrasonics Symposium, pp.329-332, 2005.

[13] Optics of thin films, Z.Knittl, John Wiley \& Sons, 1976.

[14] GU Pei-fu,Zheng Zhen-rong, "Design of non-polarizing thin film edge filters" Journal of Zhejiang University Science A,2006 7(6),pp.1037-1040.

[15] Comsol Multiphysics, version 3.5, 2009 (www.comsol.com)

[16] G.G.Fattinger, "BAW resonators design considerations - An Overview," Proc. IEEE Frequency Control Symposium, pp.762-767, 2008. 